\title{
UNIVERSITYOF BIRMINGHAM

\section{Prospective associations between cardiovascular reactions to acute psychological stress and change in physical disability in a large community sample.}

\author{
Phillips, Anna; Der, G; Shipton, D; Benzeval, M
}

DOI:

10.1016/j.ijpsycho.2011.08.002

\section{Document Version}

Peer reviewed version

Citation for published version (Harvard):

Phillips, A, Der, G, Shipton, D \& Benzeval, M 2011, 'Prospective associations between cardiovascular reactions to acute psychological stress and change in physical disability in a large community sample.', International journal of psychophysiology : official journal of the International Organization of Psychophysiology, vol. 81, no. 3, pp. 332-7. https://doi.org/10.1016/j.ijpsycho.2011.08.002

Link to publication on Research at Birmingham portal

Unless a licence is specified above, all rights (including copyright and moral rights) in this document are retained by the authors and/or the copyright holders. The express permission of the copyright holder must be obtained for any use of this material other than for purposes permitted by law.

- Users may freely distribute the URL that is used to identify this publication.

- Users may download and/or print one copy of the publication from the University of Birmingham research portal for the purpose of private study or non-commercial research.

- User may use extracts from the document in line with the concept of 'fair dealing' under the Copyright, Designs and Patents Act 1988 (?)

-Users may not further distribute the material nor use it for the purposes of commercial gain.

Where a licence is displayed above, please note the terms and conditions of the licence govern your use of this document.

When citing, please reference the published version.

Take down policy

While the University of Birmingham exercises care and attention in making items available there are rare occasions when an item has been uploaded in error or has been deemed to be commercially or otherwise sensitive.

If you believe that this is the case for this document, please contact UBIRA@lists.bham.ac.uk providing details and we will remove access to the work immediately and investigate.
} 
Phillips, A.C., Der, G., Shipton, D., \& Benzeval, M. (2011). Prospective associations between cardiovascular reactions to acute psychological stress and change in physical disability in a large community sample. International Journal of Psychophysiology, 81, 332-337. http://dx.doi.org/10.1016/j.ijpsycho.2011.08.002

Prospective associations between cardiovascular reactions to acute psychological stress and change in physical disability in a large community sample

Running Head: Disability and reactivity

\author{
Anna C. Phillips ${ }^{1}$ \\ Geoff Der ${ }^{2}$ \\ Deborah Shipton $^{2}$ \\ Michaela Benzeval ${ }^{2}$ \\ ${ }^{1}$ School of Sport and Exercise Sciences, University of Birmingham, Birmingham, \\ England \\ ${ }^{2}$ MRC Social and Public Health Sciences Unit, University of Glasgow, Glasgow, \\ Scotland
}

Number of text pages:

Number of tables:

Number of figures:

Address correspondence to: Anna C. Phillips, PhD, School of Sport and Exercise

Sciences, University of Birmingham, Birmingham B15 2TT, England, UK. Tel: (00) 44

121414 4398, Fax: (00) 44121414 4121. E-mail: A.C.Phillips@ bham.ac.uk 
Phillips, A.C., Der, G., Shipton, D., \& Benzeval, M. (2011). Prospective associations between cardiovascular reactions to acute psychological stress and change in physical disability in a large community sample. International Journal of Psychophysiology, 81, 332-337. http://dx.doi.org/10.1016/j.ijpsycho.2011.08.002

\begin{abstract}
Exaggerated haemodynamic reactions to acute psychological stress have been implicated in cardiovascular disease outcomes, while lower reactions have been considered benign. This study examined, in a large cohort, the prospective associations between stress reactivity and physical disability. Blood pressure and pulse rate were measured at rest and in response to a stress task. Physical disability was assessed using the OPCS survey of disability at baseline and five years later. Heart rate reactivity was negatively associated with change in physical disability over time, such that those with lower heart rate reactivity were more likely to deteriorate over the following five years. These effects remained significant following adjustment for a number of confounding variables. These data give further support to the recent argument that for some health outcomes, lower or blunted cardiovascular stress reactivity is not necessarily protective.

Key words: Acute psychological stress; Blood pressure; Cardiovascular reactivity; Disability; Heart rate;
\end{abstract}


Phillips, A.C., Der, G., Shipton, D., \& Benzeval, M. (2011). Prospective associations between cardiovascular reactions to acute psychological stress and change in physical disability in a large community sample. International Journal of Psychophysiology, 81, 332-337. http://dx.doi.org/10.1016/j.ijpsycho.2011.08.002

\section{Introduction}

The reactivity hypothesis, as originally conceptualised, proposed that large magnitude cardiovascular reactions to acute psychological stress exposures increase the risk of developing hypertension (Obrist, 1981). Studies testing the hypothesis have reliably shown that high reactivity is associated prospectively with hypertension and/or an upward shift in blood pressure (【Carroll et al., 2001], [Markovitz et al., 1998$]$ and [Treiber et al., 19971). The risk associated with higher cardiovascular reactivity has also been shown to extend to other cardiovascular outcomes including carotid atherosclerosis and increased left ventricular mass/cardiac hypertrophy, e.g., ([Everson et al., 1997], [Georgiades et al., 1997], [Kamarck et al., 1997], [Matthews et al., 2006] and [Treiber et al., 2003]).

An obvious corollary of the reactivity hypothesis is that low reactivity during acute stress is an adaptive response and protective against cardiovascular disease. By extension, then, low reactivity would be expected to characterise more positive psychological and behavioural factors implicated in mitigating cardiovascular disease risk. However, findings emerging recently suggest that negative characteristics or behaviours might not always be associated with higher cardiovascular reactivity. For example, in the Twenty07 study, a longitudinal cohort based in and around Glasgow, socio-economic status was positively associated with acute stress reactivity such that those from manual occupational households had lower cardiovascular responses to acute stress than those from non-manual households (Carroll et al., 2000). Smokers also demonstrated blunted reactivity relative to non-smokers or ex-smokers (Phillips et al., 2009c). Similarly, it might be expected that individuals suffering a greater burden of stressful life events would show exaggerated cardiovascular reactivity to acute stress, but again in the Twenty-07 study, middle-aged and older adults with higher disruptiveness ratings for the stressful events they had experienced in the past two years exhibited blunted systolic blood pressure reactions to acute mental stress (Carroll et al., 2005). Likewise, in younger adults, the total number of life events, including work and family events, and the number of personal life events were negatively associated with systolic blood pressure and pulse rate reactions to acute stress (Phillips et al., 2005). 
Phillips, A.C., Der, G., Shipton, D., \& Benzeval, M. (2011). Prospective associations between cardiovascular reactions to acute psychological stress and change in physical disability in a large community sample. International Journal of Psychophysiology, 81, 332-337. http://dx.doi.org/10.1016/j.ijpsycho.2011.08.002

More recently, prospective associations have been demonstrated between blunted cardiovascular reactivity and a range of negative health outcomes in the Twenty-07 study, including an increase in depressive symptoms ([Carroll et al., 2007] and [Phillips et al., 20101), increased likelihood of developing obesity (Carroll et al., 2008), and a decline in self-reported health (Phillips et al., 2009b). For a review, see Phillips, (2011b).

Such seemingly anomalous results could not readily be attributed to reduced attention to and disengagement from the stress task, as all these associations withstood adjustment for stress task performance. In addition, associations between low reactivity and adverse health and behavioural outcomes are not confined to the Twenty-07 dataset. For example, in a study of depression and reactivity to a public-speaking stress task in over 100 coronary artery disease patients, higher depressive symptom scores were associated with lower, not higher, reactivity (York et al., 2007). Similarly, individuals with sub-clinical depression levels displayed blunted cardiovascular responses to tasks associated with the consequences of punishment and reward in comparison to those with lower depression (Brinkmann et al., 2009). Further, in a cross-sectional study of men and women aged between 55 and 60 years, those with a diagnosis of depression or anxiety and those with mild to severe depression or anxiety symptoms had lower blood pressure, heart rate, and cortisol reactivity to Stroop, mirror tracing, and speech stress tasks than those without a diagnosis or symptoms of depression or anxiety (de Rooij et al., 2010). The findings for self-reported health have also been replicated in this Dutch cohort study, showing that those with larger cardiovascular reactions to acute psychological stress reported better health than those with small reactions; the same held true for cortisol reactivity (De Rooij and Roseboom, 2010). Blunted cardiovascular reactivity has been observed in female smokers regardless of whether they were wearing a nicotine replacement patch or not (Girdler et al., 1997) and among smokers regardless of whether they continued to smoke or abstained during testing (al'Absi et al., 2003). In fact cardiovascular and cortisol hyporesponsiveness predicted relapse among smokers who had quit, suggesting prognostic significance ([al'Absi, 2006] and [al'Absi et al., 2005]). Similarly, alcohol addiction was found to relate to blunted cardiovascular and cortisol reactivity ( $[$ Lovallo et al., 2000] and [Panknin et al., 2002]). In the laboratory, blunted cardiovascular reactivity has 
Phillips, A.C., Der, G., Shipton, D., \& Benzeval, M. (2011). Prospective associations between cardiovascular reactions to acute psychological stress and change in physical disability in a large community sample. International Journal of Psychophysiology, 81, 332-337. http://dx.doi.org/10.1016/j.ijpsycho.2011.08.002

also been associated with poorer responses to influenza vaccination (Phillips et al., 2009a) and exercise addiction (Heaney et al., 2011).

Possible explanations for this surprising direction of findings are that, although greater reactivity is detrimental to cardiovascular outcomes, this might not be the case for all outcomes. Certainly, the animal literature would propose that reactions to acute stress can in fact be adaptive and enhancing, particularly in the instance of immune function, which when upregulated by stress improves host defence against infection (Dhabhar, 2002), which we have also provisionally demonstrated in humans as mentioned earlier regarding the response to vaccination ([Edwards et al., 2006] and [Phillips et al., 2009a]). Given that reactivity appears to have implications for health broader than originally envisaged, now appearing to extend to risk for developing psychological symptoms, obesity, and poor self-reported health in the future, it is perhaps surprising that there have been few studies examining the prospective associations between stress reactivity and future physical function or disability. As far as we are aware, only one study has investigated the association between cardiovascular reactivity and future disability. In a study of 50 patients with multiple sclerosis, those with higher cardiovascular reactivity to acute stress demonstrated a greater number of disease exacerbations and weeks of illness in the following year (Ackerman et al., 2003). This was attributed to increased sympathetic nervous system activity relating to disease activity, perhaps via greater production of Th-1 cytokines in response to stress (Ackerman et al., 2003). What is not yet known is whether individuals' responses to acute stress relate to a decrease in general physical disability associated with a range of diseases and ageing.

The present analyses of data from a large community sample of men and women allowed us to examine the issue of whether the magnitude of haemodynamic reactions to a standard mental stress task was linked to the change in physical disability prospectively over five years. In addition, the richness of the database permitted adjustment for a number of potential confounders.

\section{Materials and Methods}

\subsection{Participants}


Phillips, A.C., Der, G., Shipton, D., \& Benzeval, M. (2011). Prospective associations between cardiovascular reactions to acute psychological stress and change in physical disability in a large community sample. International Journal of Psychophysiology, 81, 332-337. http://dx.doi.org/10.1016/j.ijpsycho.2011.08.002

Data were collected as part of the West of Scotland Twenty-07 Study. Participants were all from Glasgow and surrounding areas in Scotland, and have been followed up at regular intervals since the original survey in 1987/8 (Benzeval et al., 2009). The study was designed to examine the processes that produce and maintain socio-demographic differences in health (Macintyre, 1987). The data reported here are from two age cohorts (born in the 1930s and 1950s) at the third follow-up carried out in 1995 (called baseline hereafter) and fourth follow-up conducted in 2000/04. The mean (SD) time lag between these follow-ups was 5.4 (0.88) years. Physical disability was measured at both time points. At baseline, cardiovascular reactions to an acute psychological stress task were measured ([Carroll et al., 2000] and [Carroll et al., 2003]) and reactivity data were available for 1038 participants who also provided disability information. There were 563 (54\%) women and 475 (46\%) men. Disability information was also available for 852 respondents at follow up. The sample was almost entirely Caucasian, reflecting the Westof-Scotland population from which it was drawn. Overall mean age at baseline was 51.9 $(\mathrm{SD}=9.39)$ years; $44.1(0.85)$ years for the $614(59 \%)$ respondents from the middle cohort and $63.1(0.67)$ years for the $424(41 \%)$ respondents from the oldest cohort. Local ethics committee approval was obtained for each phase of data collection and all the participants provided written informed consent at each follow-up (Benzeval et al., 2009).

\subsection{Measures}

Household occupational group was classified as manual or non-manual from the current or most recent occupation of the head of household, using the Registrar General's Classification of Occupations (OPCS, 1980). Head of household was defined as the man in couple households if he had an occupation, otherwise it was the woman. Participants were classified as current smokers or not by their response to a simple question: "do you ever smoke tobacco now? I am thinking of a pipe, cigars, and your own roll-ups as well as cigarettes you might buy". They also reported whether they were currently taking antihypertensive medication. Body mass index (BMI) was calculated from measured height and weight. Depressive symptoms were measured using the Hospital Anxiety and Depression Scale (HADS) (Zigmond and Snaith, 1983), as detailed elsewhere (Carroll et al., 2007). All these measures were taken at the baseline of the study. 
Phillips, A.C., Der, G., Shipton, D., \& Benzeval, M. (2011). Prospective associations between cardiovascular reactions to acute psychological stress and change in physical disability in a large community sample. International Journal of Psychophysiology, 81, 332-337. http://dx.doi.org/10.1016/j.ijpsycho.2011.08.002

\subsubsection{Physical disability scale}

Disability was assessed using the Office of Population Censuses and Surveys (OPCS) disability survey (Martin et al., 1988). The measure has been used extensively in national health surveys to map disability in the population (Grundy et al., 1999). For the present study, only the six most commonly used subscales were included (locomotion, reaching and stretching, dexterity, seeing, hearing, and continence). Typical questions are described in supplementary data (Figure S1). Each domain has a series of questions to ascertain the extent of limitation experienced by the participant, which is then assigned a severity score based on the OPCS Survey (Martin et al., 1988). (Examples of the severity score assigned to different levels of limitations on different domains are shown in Figure S2). The overall disability score is the weighted sum of the values of the three highest domain scores $[$ score $=(1 *$ worst score $)+(0.4 *$ second worst score $)+(0.3 *$ third worst score)]. Scores were calculated for baseline and later follow-up.

\subsection{Procedure}

Participants were interviewed in a quiet room in their homes by trained nurses. As part of a structured interview, the aforementioned measures were taken. At baseline, participants also undertook an acute psychological stress task.

\subsubsection{Stress task}

The acute psychological stress task was the paced auditory serial addition test (PASAT), which has been shown in numerous studies to reliably perturb the cardiovascular system ([Ring et al., 2002] and [Winzer et al., 1999]) and to demonstrate good test-retest reliability (Willemsen et al., 1998). Participants were presented, via audiotape, with a series of single digit numbers and had to add sequential number pairs while retaining the second of the pair in memory for addition to the next number presented, and so on throughout the series. Answers were given orally and, if participants faltered, they were prompted to begin again with the next number pair. The number of correct answers was recorded as a measure of performance. The first sequence of 30 numbers was presented at a rate of one every $4 \mathrm{~s}$, and the second sequence of 30 at one every $2 \mathrm{~s}$. The complete task took $3 \mathrm{~min}, 2 \mathrm{~min}$ for the slower and $1 \mathrm{~min}$ for the faster sequence. A brief practice was given to ensure that participants understood the demands of the task. Only those who 
Phillips, A.C., Der, G., Shipton, D., \& Benzeval, M. (2011). Prospective associations between cardiovascular reactions to acute psychological stress and change in physical disability in a large community sample. International Journal of Psychophysiology, 81, 332-337. http://dx.doi.org/10.1016/j.ijpsycho.2011.08.002

registered a score on the PASAT were included in the analyses. Out of a possible score of 60 , the mean score was $40.9(\mathrm{SD}=9.03)$.

\subsubsection{Blood pressure and heart rate}

Systolic blood pressure (SBP), diastolic blood pressure (DBP), and heart rate (HR) were measured by an Omron (model 705CP) semi-automatic sphygmomanometer recommended by the European Society of Hypertension (O'Brien et al., 2001). Following the interview, (at least an hour), there was then a formal 5-minute period of relaxed sitting, at the end of which a resting baseline reading of SBP, DBP, and HR was taken. Task instructions were then given, followed by the brief practice. Two further SBP, DBP, and HR readings were taken during the task, the first initiated $20 \mathrm{~s}$ into the task (during the slower sequence of numbers), and the second initiated $110 \mathrm{~s}$ later (at the same point during the faster sequence). For all readings, the nurses ensured that the participant's elbow and forearm rested comfortably on a table at heart level. The two task readings were averaged and the resting baseline value subsequently subtracted from the resultant average task value to yield reactivity measures for SBP, DBP, and HR for each participant.

\subsection{Statistical analyses}

Repeated measures ANOVAs, comparing baseline and mean task value, were undertaken to confirm that the PASAT perturbed cardiovascular activity. The relationship between reactivity (the difference between the mean task and the baseline value) and concurrent disability score at baseline was analysed through linear regression. The association between reactivity and change in disability status over time was analysed by linear regression, with $\mathrm{SBP}, \mathrm{DBP}$, and $\mathrm{HR}$ reactivity as the independent variable, disability score at follow-up as the dependent variable; cardiovascular levels and disability score at baseline were entered as covariates at step 1 of the models: the latter to examine the change in disability over time. Where significant effects emerged throughout, further regressions were undertaken that, in addition to resting cardiovascular level and earlier disability score, also adjusted for socio-demographic characteristics (age, sex, occupational group) at step 1 of the models (Carroll et al., 2000). Finally, significant models were repeated with separate adjustment for other potential confounders measured 
Phillips, A.C., Der, G., Shipton, D., \& Benzeval, M. (2011). Prospective associations between cardiovascular reactions to acute psychological stress and change in physical disability in a large community sample. International Journal of Psychophysiology, 81, 332-337. http://dx.doi.org/10.1016/j.ijpsycho.2011.08.002

at baseline previously found to be associated with reactivity in this sample (antihypertensive medication status, smoking status, BMI, PASAT performance score, and depressive symptoms) by entering these separately at step 1 of each model ( [Carroll et al., 2007], [Carroll et al., 2008] and [Phillips et al., 2009c]). Standardised regression coefficients $(\beta)$ and $\Delta \mathrm{R}^{2}$ are given throughout as measures of effect size.

\section{Results}

Of the 852 with reactivity data and disability information at both measurement points, there were two distinct age cohorts; 518 (61\%) ageing from 44 to 50 year olds, and 334 (39\%) ageing from 63 to 69 year olds. Across the two age cohorts, 393 (46\%) were men and 459 (54\%) were women. Regarding socio-economic status, 393 (46\%) were from manual and 456 (54\%) from non-manual occupation households. Household occupational group data were not available for three participants. The mean baseline BMI of the sample was $26.3(4.24) \mathrm{kg} / \mathrm{m}^{2}$.

\subsection{Physical disability}

The socio-demographic breakdown of disability score at each follow-up is presented in Table 1. Those in the older cohort $(p<.001)$, women $(p \leq .001)$, and those from manual occupational households $(p<.001)$ registered higher disability scores at both follow-ups. Those with higher disability scores at baseline had higher disability at followup $(p<.001)$. 
Phillips, A.C., Der, G., Shipton, D., \& Benzeval, M. (2011). Prospective associations between cardiovascular reactions to acute psychological stress and change in physical disability in a large community sample. International Journal of Psychophysiology, 81, 332-337. http://dx.doi.org/10.1016/j.ijpsycho.2011.08.002

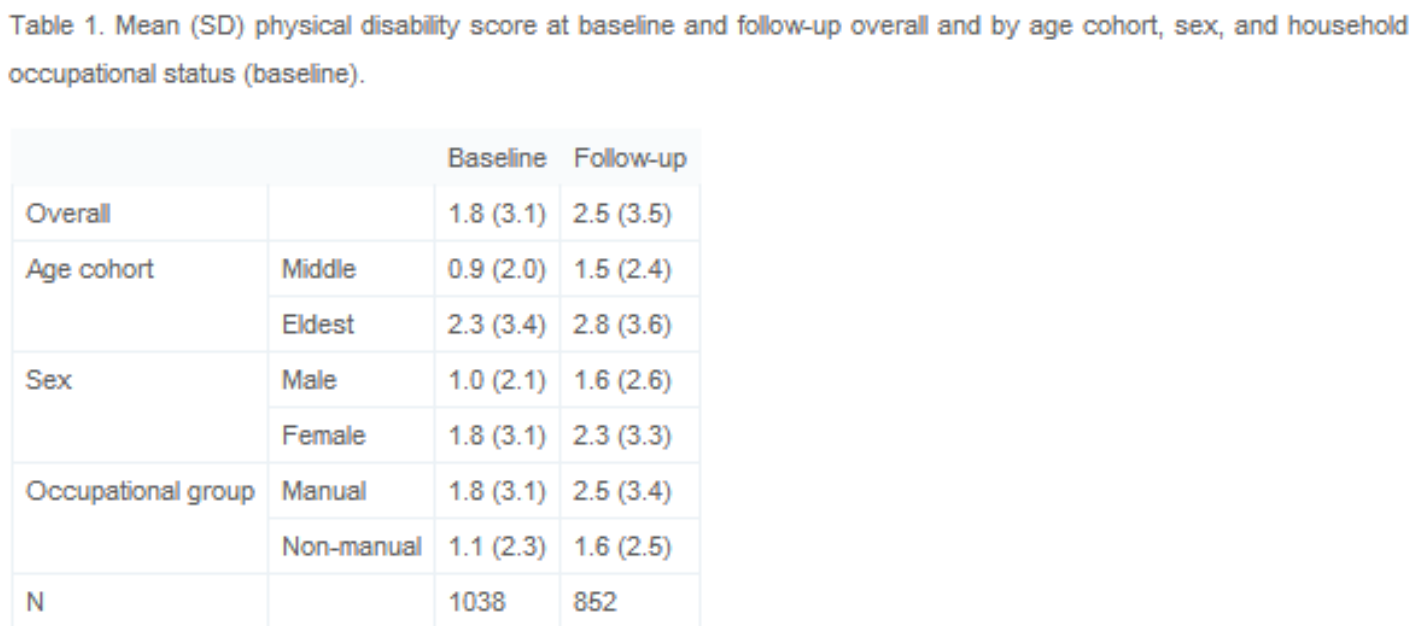

\subsection{Cardiovascular reactivity to the stress task}

The PASAT provoked significant increases in SBP, $\mathrm{F}(1,1037)=1012.82, p<.001$, $\eta^{2}=.494, \mathrm{DBP}, \mathrm{F}(1,1037)=745.09, p<.001, \eta^{2}=.418$, and HR, $\mathrm{F}(1,1037)=637.79, p<.001, \eta^{2}=.381$. We have described the socio-demographic effects on reactivity in previous reports ( [Carroll et al., 2000] and [Carroll et al., 2007]) and they are summarized in Table 2 . Briefly, HR reactivity declined with age, with the middle cohort showing higher reactivity than the eldest cohort. HR reactivity was also greater in participants from non-manual occupational status households and women were less SBP and DBP reactive than men. The mean (SD) PASAT score was 42.9 (9.46), which was significantly correlated with SBP and HR reactivity, $\mathrm{r}(1036)=.16, p<.001$, and $\mathrm{r}(1036)=.19, p<.001$, respectively. 
Phillips, A.C., Der, G., Shipton, D., \& Benzeval, M. (2011). Prospective associations between cardiovascular reactions to acute psychological stress and change in physical disability in a large community sample. International Journal of Psychophysiology, 81, 332-337. http://dx.doi.org/10.1016/j.ijpsycho.2011.08.002

Table 2. Mean (SD) SBP, DBP, and HR before and during rest and the stress task by age cohort, sex, and occupational status.

\begin{tabular}{|c|c|c|c|c|c|c|c|}
\hline \multicolumn{2}{|l|}{ Group } & \multicolumn{2}{|l|}{ SBP } & \multicolumn{2}{|l|}{ DBP } & \multicolumn{2}{|l|}{ HR } \\
\hline & & Rest & Reactivity & Rest & Reactivity & Rest & Reactivity \\
\hline \multirow[t]{2}{*}{ Age } & Middle & $\begin{array}{l}127.1 \\
(18.08)\end{array}$ & $12.3(11.42)$ & $\begin{array}{l}80.6 \\
(11.13)\end{array}$ & $7.2(7.94)$ & $\begin{array}{l}66.7 \\
(11.17)\end{array}$ & $7.8(9.92)$ \\
\hline & Eldest & $\begin{array}{l}144.4 \\
(21.68)\end{array}$ & $12.4(13.98)$ & $\begin{array}{l}83.8 \\
(11.17)\end{array}$ & $7.0(8.96)$ & $65.7(9.92)$ & $6.2(7.74)-$ \\
\hline \multirow[t]{2}{*}{ Sex } & Male & $\begin{array}{l}138.4 \\
(19.41)\end{array}$ & $14.1(12.32)$ & $\begin{array}{l}84.2 \\
(10.80)\end{array}$ & $7.6(8.05)$ & $\begin{array}{l}64.7 \\
(10.62)\end{array}$ & $7.9(9.34)$ \\
\hline & Female & $\begin{array}{l}130.5 \\
(22.29)\end{array}$ & $\begin{array}{l}10.9 \\
(12.52)=\end{array}$ & $\begin{array}{l}79.9 \\
(11.24)\end{array}$ & $6.6(8.61)$ & $\begin{array}{l}67.5 \\
(10.61)\end{array}$ & $6.5(8.90)^{*}$ \\
\hline \multirow[t]{2}{*}{$\begin{array}{l}\text { Occupational } \\
\text { group }\end{array}$} & Manual & $\begin{array}{l}135.3 \\
(22.22)\end{array}$ & $12.0(12.93)$ & $\begin{array}{l}82.2 \\
(11.50)\end{array}$ & $6.5(8.72)$ & $\begin{array}{l}66.7 \\
(11.46)\end{array}$ & $5.7(8.91)$ \\
\hline & $\begin{array}{l}\text { Non- } \\
\text { manual }\end{array}$ & $\begin{array}{l}133.0 \\
(20.52)\end{array}$ & $12.7(12.12)$ & $\begin{array}{l}81.6 \\
(10.98)\end{array}$ & $\begin{array}{l}7.6 \\
(8.00)^{*}\end{array}$ & $65.8(9.94)$ & $\begin{array}{l}8.5 \\
(9.12)=0\end{array}$ \\
\hline
\end{tabular}

"Significantly different from other group $p<.05,{ }^{* *} p<.01,{ }^{* * *} p<.001$.

\subsection{Cardiovascular reactivity and concurrent disability}

Following adjustment for resting cardiovascular levels, SBP and HR reactivity were negatively associated with concurrent disability score, such that those with lower reactivity had greater disability at baseline, seeTable 3 . DBP reactivity was not related to disability score. Following adjustment for age cohort, sex, and occupational group, SBP and HR reactivity remained significantly negatively associated with concurrent disability score, see Table 3 . These associations also remained significant following separate adjustment for antihypertensive medication usage, smoking status, and BMI. However, for HR the association with disability was attenuated after adjustment for BMI, $\beta=-.0 .02, p=.07, \Delta \mathrm{R}^{2}=.003$, and the associations for both SBP and HR reactivity were attenuated following adjustment for PASAT performance score: for SBP, $\beta=-.05, p=.11, \Delta \mathrm{R}^{2}=.002$, and $\mathrm{HR}, \beta=-.05, p=.14, \Delta \mathrm{R}^{2}=.002$, reactivity respectively. Similarly, both the association for SBP reactivity and HR reactivity were attenuated following adjustment for concurrent depression score, $\beta=-.05, p=.12$, 
Phillips, A.C., Der, G., Shipton, D., \& Benzeval, M. (2011). Prospective associations between cardiovascular reactions to acute psychological stress and change in physical disability in a large community sample. International Journal of Psychophysiology, 81, 332-337. http://dx.doi.org/10.1016/j.ijpsycho.2011.08.002

$\Delta \mathrm{R}^{2}=.002$, and $\beta=-.05, p=.14, \Delta \mathrm{R}^{2}=.002$, respectively. It is worth noting that the association with HR reactivity was attenuated to a non-significant trend in both instances.

\begin{tabular}{|c|c|c|c|}
\hline & $\beta$ & $\mathrm{p}$ & $\Delta \mathrm{R}^{2}$ \\
\hline \multicolumn{4}{|l|}{ Model 1} \\
\hline Resting SBP & .080 & .012 & \\
\hline SBP reactivity & -.082 & .010 & .006 \\
\hline Resting HR & -.032 & .316 & \\
\hline HR reactivity & -.122 & $<.001$ & .014 \\
\hline \multicolumn{4}{|l|}{ Model 2} \\
\hline Resting SBP & .006 & .867 & \\
\hline Age cohort & .247 & $<.001$ & \\
\hline Sex & .152 & $<.001$ & \\
\hline Occupational group & .113 & $<.001$ & \\
\hline SBP reactivity & -.077 & .013 & .005 \\
\hline Resting HR & -.034 & .276 & \\
\hline Age cohort & .242 & $<.001$ & \\
\hline Sex & .160 & $<.001$ & \\
\hline Occupational group & .106 & $<.001$ & \\
\hline HR reactivity & -.072 & .021 & .005 \\
\hline
\end{tabular}

3.4. Cardiovascular reactivity and change in disability over five years

Lower HR reactivity was associated with an increase in disability score controlling for prior disability. The statistics for this significant association is shown in Table 4. SBP and DBP reactivity were not significantly related to change in disability score, although the associations were in the same direction as for HR reactivity. Following adjustment for age cohort, sex, and occupational group, HR reactivity was still negatively associated with change in disability, see Table 4, Model 2, such that those with smaller HR reactions showed a greater deterioration (increase in disability score) over the subsequent five years. This association also remained significant following separate adjustment for antihypertensive medication usage, smoking status, BMI, PASAT performance score, and depressive symptoms ( $\beta=-.06$ to $-.07, p=.02$ to .04 ). Those with greater disability (higher score) at wave 3 and wave 4 had a significantly larger BMI ( $p=.005$ and .01 , 
Phillips, A.C., Der, G., Shipton, D., \& Benzeval, M. (2011). Prospective associations between cardiovascular reactions to acute psychological stress and change in physical disability in a large community sample. International Journal of Psychophysiology, 81, 332-337. http://dx.doi.org/10.1016/j.ijpsycho.2011.08.002

respectively), higher depressive symptoms scores $(p=<.001)$, and were more likely to be current smokers ( $p=<.001$ and .03 , respectively). There was no association between disability score at either measurement point and taking anti-hypertensive medication.

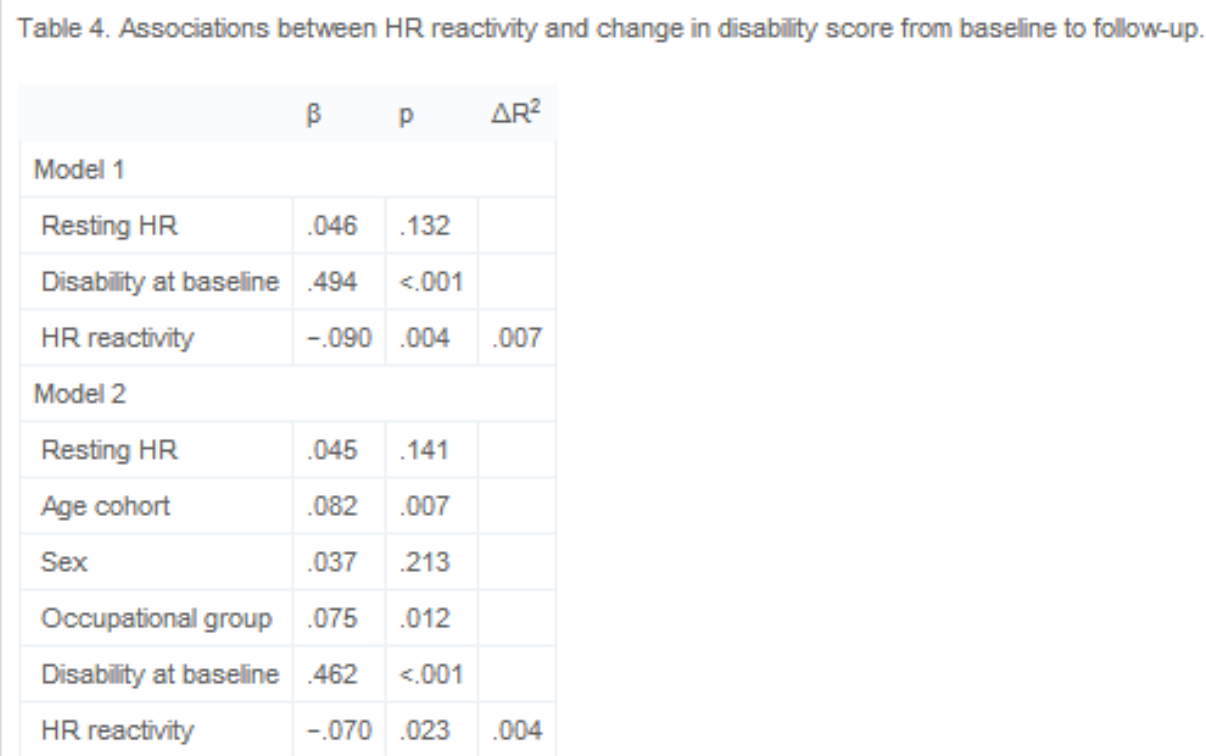

\subsection{Sensitivity analysis}

As those self-reporting poor physical function might also have lower task engagement and effort, or greater depression, a sub-analysis was run removing participants with poor physical function at baseline (above 90th percentile) leaving $\mathrm{N}=804$. The negative associations between heart rate reactivity and physical function at follow-up, adjusting for previous physical function, and age, sex, and occupational group remained significant $(p=.05)$. However, it was attenuated to a non-significant trend when further adjusted for each of the other covariates mentioned above $(p<.1$ in each case).

Further, given that there was a positive skew for the disability variables, all analyses were repeated using a log transformed version of the disability scores. The direction, magnitude, and significance of the aforementioned associations remained the same in all cases.

\section{Discussion}


Phillips, A.C., Der, G., Shipton, D., \& Benzeval, M. (2011). Prospective associations between cardiovascular reactions to acute psychological stress and change in physical disability in a large community sample. International Journal of Psychophysiology, 81, 332-337. http://dx.doi.org/10.1016/j.ijpsycho.2011.08.002

The present study found a prospective association between low HR reactivity and the deterioration in physical functional ability over time in a middle-aged and elderly sample. This association did not appear to be driven by variations in reactivity or disability with age, sex, occupational group, antihypertensive medication use, BMI, smoking status, or degree of depressive symptomatology. Neither was this association a result of poorer acute stress task performance among those with greater physical disability, as the relationship between reactivity and change in disability withstood correction for stress task performance score. There were also negative associations between SBP and HR reactivity and concurrent disability score. However, these were both attenuated following adjustment for mental stress task performance and depressive symptoms score. The direction of association observed here is consistent with previous studies of the cross-sectional and prospective relationships between blunted reactivity and a range of adverse health and behavioural indicators/outcomes including depressive symptoms, smoking, obesity, poor self-reported health, low socio-economic status, and high stressful life events levels in present dataset ([Carroll et al., 2000], [Carroll et al., 2005], [Phillips, 2011b], [Phillips et al., 2005] and [Phillips et al., 2009c]), as well as in other studies with different stress tasks ([al'Absi, 2006], [al'Absi et al., 2005], [al'Absi et al., 2003], [Brinkmann et al., 2009],[Carroll et al., 1997], [De Rooij and Roseboom, 2010], [de Rooij et al., 2010], [Girdler et al., 1997], [Lovallo et al., 2000], [Panknin et al., 2002] and [York et al., 2007]). It is also consistent with our recent findings that blunted cardiovascular reactivity is also characteristic of those exhibiting poorer responses to influenza vaccination (Phillips et al., 2009a) and those exhibiting signs of exercise addiction (Heaney et al., 2011).

It is possible that while high cardiovascular reactivity is implicated in cardiovascular health outcomes and/or diseases such as hypertension and atherosclerosis through direct pathways, blunted reactivity is associated more indirectly with the development of poor health outcomes and behaviours such as smoking, and now physical disability, perhaps indicating that such low reactions to acute stress are a marker of some form of 
Phillips, A.C., Der, G., Shipton, D., \& Benzeval, M. (2011). Prospective associations between cardiovascular reactions to acute psychological stress and change in physical disability in a large community sample. International Journal of Psychophysiology, 81, 332-337. http://dx.doi.org/10.1016/j.ijpsycho.2011.08.002

motivational dysregulation with negative implications for health and health behaviours (for a full discussion see ([Carroll et al., 2009] and [Phillips, 2011a]).

What might be the mechanisms underlying the present direction of prospective association between reactivity and disability? One possibility is that for certain health outcomes, a robust or greater response to acute stress is actually beneficial. For example, exposure to acute mental stress in our own laboratory was associated with an enhanced antibody response to the influenza vaccine in women (Edwards et al., 2006). Similarly, in animals, short-term exposure to moderate behavioural challenges has been associated with better health, mainly for immunological outcomes, and increased longevity ([Arumugam et al., 2006] and [Shevchuk and Radoja, 2007]). Poorer immune function including a decreased ability to control infectious disease is characteristic of ageing and has been found to relate to morbidity and mortality in older age (Pawelec, 2006). Indeed, one in seven deaths among the elderly is infection-related (Globerson and Effros, 2000). In addition, short-term increases in cardiovascular activity in response to stress are associated with increased numbers of immune cells in circulation and improved immune function ([Dhabhar, 2002], [Dhabhar, 2003] and [Kimura et al., 2005]) via the receptors for sympathetic nervous system neurotransmitters and neuroendocrine hormones on immune organs and various other mechanisms, see e.g., (「Cacioppo, 1994], [Dantzer and

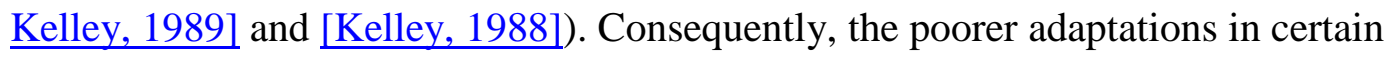
aspects of immune function, contingent with low cardiovascular reactivity, might afford an explanation of how low stress reactivity is associated with certain negative health outcomes. As the theory of allostatic load contends, an inadequate response of allostatic systems to stress can be just as dysfunctional as exaggerated activation or the failure to shut off such activation after stress, i.e. delayed recovery (McEwen, 1998). The allostatic load theory might also help to explain why certain individuals are less responsive to acute stress in the first place, as a result of desensitization from repeated or chronic environmental exposure to stress in the form of life events stress (McEwen and Stellar, 1993) or habitual unhealthy behaviours (McEwen and Seeman, 1999). The effects of such exposures and behaviours are mediated via changes to the neural plasticity of brain areas which also regulate stress reactivity (McEwen and Gianaros, 2010). 
Phillips, A.C., Der, G., Shipton, D., \& Benzeval, M. (2011). Prospective associations between cardiovascular reactions to acute psychological stress and change in physical disability in a large community sample. International Journal of Psychophysiology, 81, 332-337. http://dx.doi.org/10.1016/j.ijpsycho.2011.08.002

The present study is not without limitations. First, we measured only blood pressure and HR. It could have proved instructive to have the sort of comprehensive assessment of haemodynamics afforded by impedance cardiography. However, the decision to test participants in their own homes and the size of the sample precluded the use of this. Second, determining causality is impossible from observational data, even with prospective data, it is difficult and confounding is always a potential problem (Christenfeld et al., 2004). However, we did adjust statistically for a broad range of possible confounders, including those we have previously found to be related to reactivity. Nevertheless, residual confounding as a consequence of poorly measured or un-measured variables cannot be wholly discounted. Third, we had to rely on performance score as our measure of task engagement rather than having a measure of task stressfulness. Although this seems reasonable, in retrospect it might have proved useful to have included self-report measures of stress task impact, although studies in our own laboratory have shown that cardiovascular reactions to stress do not necessarily correlate with perceived task stressfulness ([Heaney et al., 2011] and [Phillips et al., 2009d]). Fourth, the physical disability was measured by self-report rather than objectively, and thus might be susceptible to confounding with depressive feelings and a lack of motivation to engage with the task. Indeed, the cross-sectional associations with HR and SBP reactivity were attenuated following adjustment for depressive symptoms and PASAT performance scores, suggesting that blunted reactivity among those with concurrent disability is affected by task engagement and feelings of depression. However, that the prospective associations remained significant following adjustment for these variables, similar to our previous findings, suggests that the association of blunted reactivity with an increase in disability over time is not attributable to lower task engagement or depressive symptoms among those who report higher levels of physical disability. Further, others have shown that scores on the self-report OPCS classification of disability correlates strongly with other measures of disability, such as the Barthel Index (Wellwood et al., 1995), with activities of daily living measures (Kelly and Jessop, 1996). An additional limitation is that we analysed the disability composite scores only, thus limiting understanding of the potential mechanisms underlying our associations. 
Phillips, A.C., Der, G., Shipton, D., \& Benzeval, M. (2011). Prospective associations between cardiovascular reactions to acute psychological stress and change in physical disability in a large community sample. International Journal of Psychophysiology, 81, 332-337. http://dx.doi.org/10.1016/j.ijpsycho.2011.08.002

Further, the effect sizes for the results presented earlier were small by conventional standards. However, the present effects are of the same order as the positive associations between cardiovascular reactivity and future blood pressure status in this sample (Carroll et al., 2003) and others (e.g. (〔Carroll et al., 1995], [Carroll et al., 2001] and [Markovitz et al., 1998]) and between risk factors such as BMI and smoking and change in physical disability in this sample. This suggests that, although small and unlikely to be a target for intervention, reactivity could be an important and simple marker of an underlying issue which is predictive of the decline in health over time. Finally, this sample is largely Caucasian, and thus the results may not be able to be generalised to populations of other ethnicities. However, the inclusion of both middle-aged and older adults, males and females, and those from different socio-economic groups improve the ability to generalise these findings to other Caucasian populations.

In conclusion, a prospective negative association between HR reactivity and the change in physical disability over time has been demonstrated in a middle-aged and elderly sample. This might reflect the negative impact of allostatic load and consequent blunted reactivity on non-cardiovascular health outcomes, including alterations in immune function. Future longitudinal studies would benefit from the inclusion of a variety of indices of the function of stress responsive systems including stress hormones, measures of immune function, and specific disease outcomes to help elucidate more clearly the mechanisms between lower cardiovascular reactions to stress and future physical disability. 